\title{
DEVELOPMENT OF MULTIMEDIA MODULE ON DOMESTIC WATER
}

\section{SANITATION MANAGEMENT}

\author{
A. LALITHA ${ }^{1} \&$ R. NEELA RANI ${ }^{2}$ \\ ${ }^{I}$ M.Sc Student, Department of Home Science Extension and communication management, Hyderabad, Professor \\ Jayashankar, Telangana State, Agricultural University, Andhra Pradesh, India. \\ ${ }^{2}$ Associate Professor, Department of Home Science Extension and communication management, Hyderabad, \\ Professor, Jayashankar, Telangana State, Agricultural University, Andhra Pradesh, India.
}

\begin{abstract}
A present study was conducted on developing multimedia module on domestic water sanitation management. An experimental research design was selected. A total of thirty experts from the faculty of home science were selected for conducting a beta test for quality assurance of the developed multimedia module. The scorecard was developed for data collection. The mean score was used for data analysis. The results of the study revealed that for the development of the multimedia module, content was collected and identified the issues and given to the selected experts along with a checklist for selecting key issues. Content for identifying tribal domestic water, sanitation management was translated into a vernacular language i.e. Telugu. Outlines, flow charts and storyboards were prepared for the development of the multimedia module. Using a page based authoring tool, i.e., PowerPoint multimedia module was developed. Beta testing was done for quality assurance by the experts.
\end{abstract}

KEYWORDS: Experimental Research, Multimedia Module, Beta Testing, Power Point, Water Sanitation \& Story Board

Received: Dec 30, 2017; Accepted: Jan 20, 2018; Published: Feb 12, 2018; Paper Id.: IJASRFEB201824

\section{INTRODUCTION}

Modern educational technology envisages multimedia technology to develop an effective presentation of contents. The development of these multimedia technologies offers new ways for learning which can take place in formal and informal education. It creates teaching situation, through the multi-directional sensory system to stimulate the learners' interest in learning, thereby strengthens the memory, it is capable of enhancing learning and teaching at the same time. Multimedia provides integrated service of seeing, hearing and involving. Multimedia is a means to communicate information in both an effective and cognitive manner.

In view of the above circumstances, the present study was proposed for the development of multimedia module for effective tribal domestic water sanitation using computer technology. In this study, the multimedia module was designed for an illiterate tribal population in order to create awareness and improve knowledge on water sanitation issues like the importance of safe drinking water, water sanitation practices, water purification methods, signs \& symptoms and prevention \& control of water borne diseases, waste water management methods etc. The developed model may provide a model for a tailored program offering specific health information.

Several studies also reported that multimedia interventions and animated modules are successful in improving knowledge, and changing the attitude of the learners. So the present study is formulated to know how 
developed multimedia modules on tribal domestic water sanitation incorporating with different media formats such as text, images, animation and video are effective with the following objectives.

Sriram and Maheswari (2013) conducted studies on Integrated Communication Strategy for Creating Awareness on Sanitation and Hygiene Behavior Change. It was analyzed the awareness level on sanitation and the effectiveness of the prevailing Information, Education and Communication (IEC) approach among rural Indians. Focus Group Discussion and Survey methods were used to analyze the awareness level and effectiveness of IEC among the rural people and also a new Integrated Communication Strategy (ICS) to improve the situation.

WHO (World health organization) (2008) in their study Guidance on communication with respect to safe drinking water and household hygiene reported that as part of the communication strategy, they developed the national hygiene map, a three-page leaflet on cardboard with pictures, graphics and limited text. It was predominantly on safe water systems, with photographs of chlorinating water, how to store it and other methods of making sure drinking water was safe, boiling, but it predominantly focussed on safe water supply. And also they used local radio to get some music or a drama in between the message to break it all up every fifteen minutes.

\section{METHODOLOGY}

For the study, based upon the nature of the research problem and objectives of the present study, experimental research design was selected.

A total of thirty experts from the faculty of home science were selected for conducting a beta test for quality assurance of the developed multimedia module. The scorecard was developed for the study to gather the information from the respondents.

The mean scores were calculated to select the relevant tribal water sanitation issues for development of multimedia module and to assess the content effectiveness of the module.

\section{RESULTS AND DISCUSSIONS}

In the present study, for selection of the suitable content for development of multimedia module on tribal domestic water sanitation, key issues were collected and compiled and then were given to the selected experts with a check list on two point continuum "Required" \& "Not required". The content which was mostly stated as required by the experts was identified and incorporated in the module. The content which was stated by the majority as "Not required" was discarded.

Based on the suggestions of the experts, the content was developed in various forms like text, images, videos etc. Maximum effort was taken in the collection of vernacular words for development of the module. Content for identified tribal domestic water, sanitation management were translated into a vernacular language, i.e. telugu for module development and finalized for the production of multimedia modules. Relevant images and graphics and videos were also collected from various sources.

Based on the content collection, outlines were prepared for the development of the multimedia module. Flow charts and storyboards were also prepared incorporating multimedia elements. 
For the multimedia module development, a page based authoring tool - Power point software was used as it has many user friendly features. According to (Ron Leunissen, 2013), Power Point has got several strong points over other $e$ Learning authoring tools. E.g. PowerPoint has got extended features for shaping and formatting objects. Shadows, mirror-effect, fill colors, see-through-colors, layers, it can all be done easily within this one authoring tool. No need for another imaging tool. Because of this shaping ability, PowerPoint is the only authoring tool thus far, which can produce hot spots in any shape you want. All building blocks of multimedia like video, audio, text, animation and interactivity can be incorporated in powerpoint.

Beta testing was done for quality assurance that the developed module was given to the selected thirty experts for analyzing the content effectiveness in terms of content analysis and module analysis. Based on the suggestions, the module was modified and used in the dissemination process in the tribal area. The following flow chart explains the process of development.

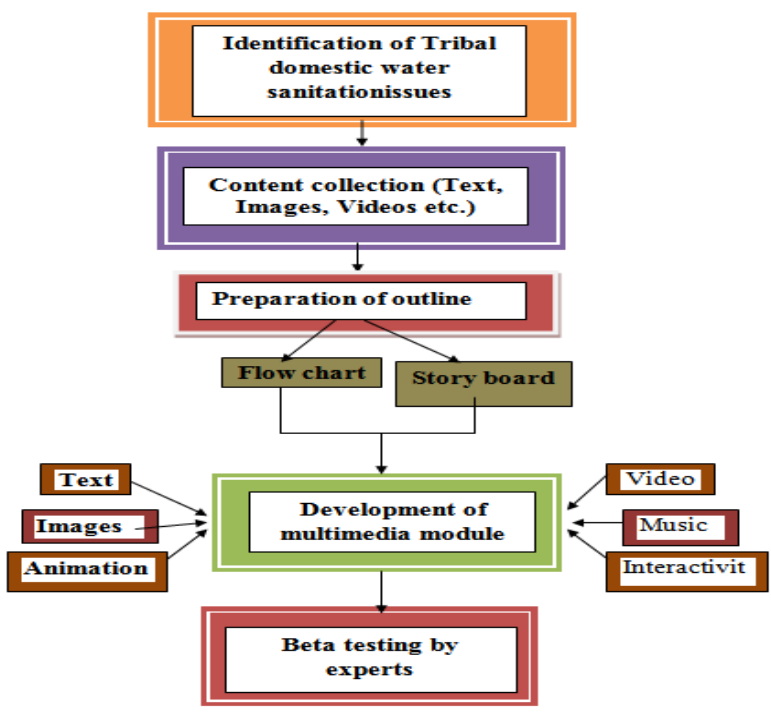

Figure 1: Process of Development of Multimedia Module on Tribal Domestic Water Sanitation

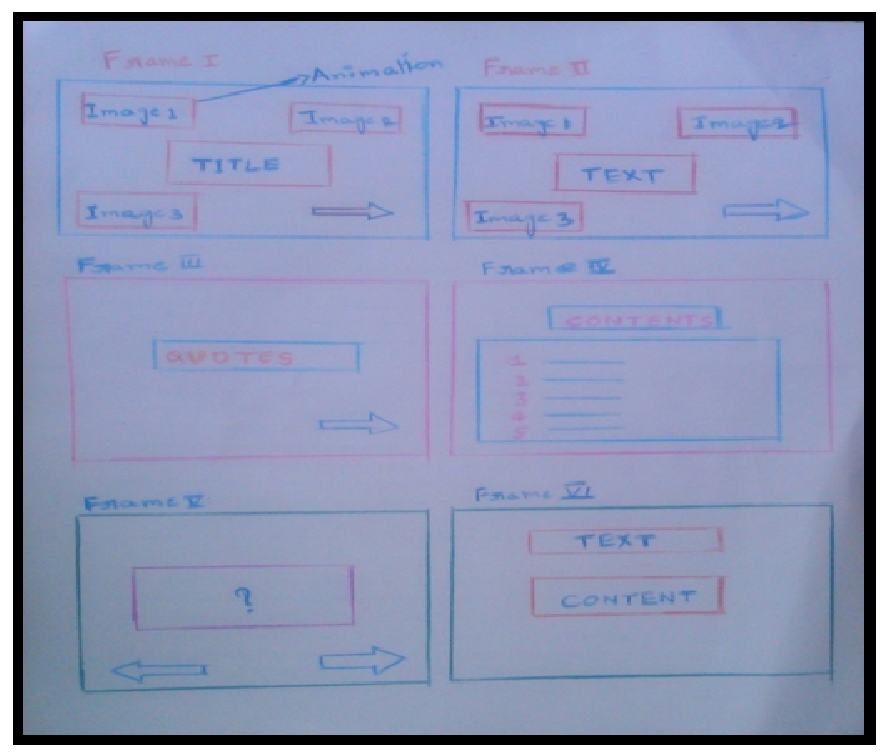

Figure 2: Story Board for Development of Multimedia Module 


\section{CONCLUSIONS}

Finally, it was concluded that development of the multimedia module, content was collected and identified the issues and given to the selected experts along with a checklist for selecting key issues. Content for identifying tribal domestic water, sanitation management were translated into a vernacular language i.e. Telugu. Outlines, flow charts and storyboards were prepared for the development of the multimedia module. Using a page based authoring tool, i.e., PowerPoint multimedia module was developed. Beta testing was done for quality assurance by the experts.

\section{REFERENCES}

1. Sriram,A and Maheswari, U.2013 Integrated Communication Strategy for Creating

2. Awareness on Sanitation and Hygiene Behavior Change the International journalof communication and health.54-59.

3. A. Lalitha \& A. Swarna Latha, A Study on Profile Characteristics of Tribal Women on Domestic Water Sanitation Practicesin Adilabad District, International Journal of Environment, Ecology, Family and Urban Studies (IJEEFUS), Volume 7, Issue 1, January - February 2017, pp. 47-52

4. World health organization(WHO). 2008. Report on Guidance on communication with respect to safe drinking water and household hygiene. 